\title{
Glottopol
}

Revue de sociolinguistique en ligne

Varia - Liberté, égalité, diversité !

\section{Usages du numérique et illectronisme : nouvelles tâches pour le lecteur-scripteur, nouveaux enjeux d'apprentissage}

\section{Élise Gandon}

\section{(2) OpenEdition \\ Journals \\ Édition électronique \\ URL : https://journals.openedition.org/glottopol/580 \\ DOI : $10.4000 /$ glottopol.580 \\ ISSN : 1769-7425 \\ Éditeur}

Presses universitaires de Rouen et du Havre

\section{Référence électronique}

Élise Gandon, «Usages du numérique et illectronisme : nouvelles tâches pour le lecteur-scripteur, nouveaux enjeux d'apprentissage », Glottopol [En ligne], 33 | 2020, mis en ligne le 01 janvier 2020, consulté le 02 octobre 2021. URL : http://journals.openedition.org/glottopol/580 ; DOI : https:// doi.org/10.4000/glottopol.580 


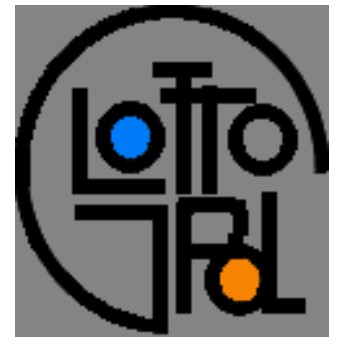

\section{GLOTTOPOL}

Revue de sociolinguistique en ligne $n^{\circ} 33$ - février 2020

Varia : Liberté, égalité, diversité !

\section{SOMMAIRE}

Hommages à Jean-Baptiste Marcellesi : À ringrazià vi, par Niculau Sorba ; “Ciao Ziu ! » par Philippe Blanchet.

Clara Mortamet : Présentation du numéro : Liberté, égalité, diversité !

Manon Him-Aquilli : "Prendre la parole sans prendre le pouvoir ». La mise en registre de l'horizontalité dans les assemblées générales anarchistes/autonomes.

Siham Hocini : La formulation du désaccord dans les débats télévisés algériens : quels "effetsde-politesse "?

Pauline Rannou : Trajectoires de la surdité en France. Parents entendants d'enfants sourds : questionner la fragmentation de l'identité sourde.

Catherine Combaz-Champlaine: Les rectifications orthographiques de 1990 comme révélateurs du rapport des enseignants à l'orthographe.

Marie-Madeleine Bertucci : Éléments d'épistémologie pour l'étude des espaces ségrégués des banlieues urbaines sensibles de la région parisienne.

Élise Gandon: Usages du numérique et illectronisme: nouvelles tâches pour le lecteurscripteur, nouveaux enjeux d'apprentissage.

Marc Arabyan : Quelle langue pour la recherche neurolinguistique française?

José R. de Arellano : Le rapprochement entre l'espagnol et le portugais pendant la décennie dorée sud-américaine : une problématique de l'horizontalité supercentrale.

Adam Wilson: Normes interactionnelles globalisées et communautés de pratique discontinues : les dynamiques sociolinguistiques du tourisme international.

Mélanie Lancien : Caractérisation de la variation liée à la situation de communication : apport de l'acoustique à la phonostylistique.

Assémou Maurice Ludovic Assémou : La prononciation du français parlé en Côte d'Ivoire. 


\title{
USAGES DU NUMÉRIQUE ET ILLECTRONISME : NOUVELLES TÂCHES POUR LE LECTEUR-SCRIPTEUR, NOUVEAUX ENJEUX D'APPRENTISSAGE
}

\author{
Élise Gandon \\ Université de Lorraine, Laboratoire Atilf
}

\section{Introduction}

Le numérique s'est imposé massivement dans nos usages au quotidien ainsi que dans nos pratiques professionnelles. Le numérique ne cesse d'évoluer. Nous vivons dans une ère où tout va vite, dans une « société de l'accélération », comme le décrit Hartmut Rosa (2010). La société a changé et change encore et avec elle nos métiers et nos compétences. De nouvelles applications pour nos tablettes ou nos smartphones voient le jour chaque semaine, certaines prennent une place prépondérante dans notre quotidien pour communiquer, s'informer, apprendre, effectuer des démarches administratives, chercher un emploi... Dans de nombreuses situations, nous faisons appel à un outil numérique parmi les diverses propositions qui s'offrent à nous : ordinateur, tablettes, smartphone... S'exprimer sur les réseaux sociaux ou consulter les actualités en ligne est pour nombre des français la première et la dernière activité de la journée (Portnoff, 2019). Par ailleurs, de nombreuses démarches se font uniquement sur Internet : la déclaration de revenus, l'inscription sur les listes électorales... et l'État prévoit une dématérialisation complète des démarches administratives pour 2022, impliquant en même temps une réduction des accueils physiques en agences. Certains de nos usages numériques sont donc contraints. Néanmoins, lorsqu'on les maitrise, ils peuvent offrir l'opportunité aux individus de s'informer, de se former, de participer à des faits de société, de communiquer.

Mais qu'en est-il des personnes qui sont en difficulté avec ces outils et des publics «non autonomes numériquement » qui sont mis à l'écart de fait par le «traitement à distance » de la relation et empêchés matériellement dans l'accès à leurs droits (Mazet, 2019) ? Nous tenterons de démontrer que l'utilisation des outils numériques requiert des compétences particulières mais également que les outils numériques peuvent permettre de développer la maitrise de l'écrit.

Nous tenterons de proposer une définition du terme illectronisme en présentant le public concerné, les aptitudes que requiert l'utilisation des outils numériques ainsi que les difficultés vécues par les personnes qui ne les maitrisent pas, ou pas suffisamment. Dans un second temps, nous développerons les tâches mobilisées dans les activités de lecture et d'écriture numériques. Enfin, nous discuterons des enjeux liés à l'acquisition des usages numériques et de l'apport des outils numériques dans l'apprentissage et nous présenterons un projet innovant : un dispositif 
de formation permettant de travailler à la fois l'écrit et les usages du numérique à destination de personnes salariées en situation d'illettrisme et d'illectronisme dans la métropole de Lyon.

\section{Qu'est-ce que l'illectronisme? Qui sont les personnes en situation d'illectronisme? Quelles difficultés rencontrent-elles?}

\section{Une proposition de définition}

Un néologisme est apparu durant cette dernière décennie : l'illectronisme. Il peut se définir comme le manque d'acquis nécessaires à l'utilisation des outils numériques. Le terme illectronisme n'est pas le seul à être utilisé. On trouve en effet une variété pléthorique d'expressions pour aborder les usages et compétences liées au numérique ou au contraire le fait de ne pas les maitriser: accès à l'e-lettrisme ${ }^{1}$, littératie numérique ou informatique, alphabétisme numérique. Les anglophones utilisent le terme de digital literacy, digital illiteracy ou computer illiteracy. Selon l'OCDE, le terme de "littératie numérique » (OCDE, 2000) pourrait se définir comme étant $l^{\prime}$ ' « aptitude à comprendre et à utiliser le numérique dans la vie courante, à la maison, au travail et dans la collectivité en vue d'atteindre des buts personnels et d'étendre ses connaissances et ses capacités » (cité par Bros, 2015 : 76).

Le terme illectronisme transpose le concept d'illettrisme au domaine du numérique. Bien que la notion d'illectronisme ne concerne pas forcément les personnes en situation d'illettrisme, il est fortement lié à l'illettrisme, $90 \%$ du contenu du Web étant textuel. Les personnes qui ne maitrisent pas les compétences de base pour lire, écrire et compter, sont bien souvent confrontées à des difficultés face à l'omniprésence des technologies numériques dans notre quotidien : suivi d'un dossier au Pôle Emploi, prestation familiale, déclaration fiscale, suivi de dossier médical, etc.

Afin de présenter le profil des personnes qui sont, selon cette définition, en situation d'illectronisme, nous nous appuyons sur deux études menées en 2018 : une première étude commandée par le syndicat de la presse sociale auprès de l'organisme $\mathrm{CSA}^{2}$ Research et une recherche menée par le CREDOC ${ }^{3}$.

Selon les chiffres de l'enquête CSA, environ $23 \%$ des Français ne sont « pas à l'aise avec le numérique » et déclarent «ne jamais naviguer sur Internet ou bien difficilement». Les chiffres sont de $58 \%$ chez les personnes de 70 ans et plus. En effet, les personnes âgées sont bien souvent complètement éloignées des réalités du numérique. $67 \%$ des personnes de 70 ans et plus ne possèdent pas de matériels pour se connecter à Internet et plus d'un tiers des personnes de 70 ans et plus ne disposent pas du tout de connexion à Internet. En effet, même si $89 \%$ des personnes interrogées possèdent au moins un équipement leur permettant d'accéder à Internet, les difficultés sont fortement liées à l'acquisition du matériel.

L'étude présente également un certain nombre de personnes nommées «abandonnistes ». Ces personnes sont définies comme ayant déjà renoncé, dans les douze derniers mois, à effectuer une action sur Internet (achat en ligne, demande d'aide sociale...) parce qu'elle nécessitait l'utilisation d'Internet. Ces personnes représentent environ un tiers des Français $(32 \%)$.

Parmi les personnes qui n'ont pas de connexion à Internet à leur domicile, certaines se rendent dans d'autres lieux où elles peuvent y avoir accès, comme par exemple chez un proche, au travail, dans une médiathèque ou un Cybercafé. Seulement $4 \%$ d'entre eux utilisent les

\footnotetext{
${ }^{1}$ Frédérique Bros (2015) Devenir e-lettré : quels leviers et voies d'accès à l'écrit à l'heure de la littératie numérique?

${ }^{2}$ Consumer Science and Analytics (CSA Research : institut de sondages).

${ }^{3}$ L'enquête du CREDOC (Centre de recherche pour l'étude et l'observation des conditions de vie) est insérée par l'ARCEP, le CGE et l'Agence du Numérique dans l'enquête sur les «Conditions de vie et les Aspirations ».
} 
services des administrations qui mettent à disposition des ordinateurs avec accès internet. Néanmoins, de manière générale, les personnes qui ne possèdent pas de connexion à Internet se connectent peu ailleurs. Ils n'utilisent tout simplement pas Internet.

Les réflexions sur l'illectronisme ou sur la littératie numérique aboutissent souvent à réfléchir aux usages du numérique sous l'angle de connaissances et de compétences à acquérir (Mazet, 2019; Connolly \& McGuinness, 2018; Bros, 2015). Nous distinguerons trois compétences principales. Tout d'abord, la compétence informatique, ou instrumentale, qui concerne les aspects techniques et pratiques des outils et du matériel. La deuxième compétence serait la compétence communicationnelle qui consiste à savoir communiquer, c'est-à-dire à utiliser les différents outils de communication du numérique mais qui réside également dans la maîtrise d'un certain nombre de codes sociaux. Enfin, la compétence médiatique (ou informationnelle) relève de l'aptitude à se repérer au sein des ressources numériques, à vérifier la fiabilité du contenu dans le cadre d'une recherche d'informations, compétence que Pierre Fastrez (2012) nomme également la littératie médiatique.

\section{La compétence informatique ou instrumentale}

La compétence informatique concerne les aptitudes techniques dans la manipulation des outils numériques. L'utilisation de l'ordinateur et ses fonctions de base en sont un exemple classique mais la compétence informatique est également liée à la manipulation des équipements et des différents périphériques. Utiliser un scanner, une imprimante ou un espace de stockage est aujourd'hui une action que nous pouvons avoir à faire au quotidien. Pour transformer des documents papiers en fichier numérique, l'usager peut utiliser un scanner et ainsi enregistrer ses fichiers, puis éventuellement les compresser et ensuite utiliser un service de transfert de fichiers ou de partage de fichiers, sur un cloud selon le poids des documents...

Certaines situations nous contraignent à utiliser ces outils comme dans le cas d'une demande d'indemnisation à Pôle Emploi : pour percevoir des allocations chômage, le demandeur doit transmettre des documents numériques via son espace emploi.

\section{La compétence communicationnelle}

Depuis l'arrivée des outils numériques dans la société, une certaine catégorie de la population française communique au travail et dans la vie privée, de manière synchrone ou asynchrone, orale ou écrite, via des outils numériques multiples: messagerie électronique (Outlook...), messagerie instantanée (Messenger, Whatsapp...), vidéoconférence (Skype, Webroom...), outils collaboratifs (Slack, Padlet, Framapad...), partage de photos et de vidéos (Instagram, Snapchat, Youtube ...). La plupart de ces outils possède toutes ces fonctionnalités à la fois. Il en nait sans cesse de nouveaux. La compétence communicationnelle consiste à savoir communiquer à l'aide de ces différents outils, c'est-à-dire à être en mesure de les utiliser mais également d'en maitriser les codes sociaux. La compétence communicationnelle consiste donc par exemple à faire un bon usage de la messagerie électronique en utilisant notamment les formules de politesse adaptée, en adaptant l'utilisation des émoticônes, en maniant avec pertinence les champs lors d'un envoi de mail groupé (À, CC, Cci). Dans le contexte professionnel également, les compétences numériques sont requises par les employeurs. Savoir fixer des dates de réunion via Outlook ou travailler en équipe sur un "mur collaboratif» numérique, échanger avec des fournisseurs lors d'une réunion à distance... Linguistique, sociolinguistique et numérique sont souvent étroitement liés.

Le Web peut également permettre aux individus d'avoir l'opportunité de s'exprimer, d'exprimer leur créativité et de la partager, de créer des groupes sociaux, d'échanger, de participer à des projets en collaboration, via les réseaux sociaux ou les sites qui diffusent des vidéos comme Youtube. 


\section{La compétence médiatique ou informationnelle}

L'une des utilisations du Web est de chercher des informations utiles au quotidien ou tout simplement de s'informer, pour apprendre. Lorsque nous effectuons ce type de recherche, nous subissons une sollicitation d'information constante. Nous devons apprendre à nous mouvoir dans ce flot d'information. Il s'agit donc d'apprendre à trier, à sélectionner des contenus sur le Web, pertinents et fiables, mais également de développer un regard critique face à ces contenus et aux réseaux sociaux. De nombreux usagers du numérique éprouvent des difficultés à se repérer au sein des ressources numériques disponibles sur le Web et à en vérifier la fiabilité. Il peut donc s'avérer nécessaire de pouvoir être formé, accompagné afin d'acquérir cette compétence (Tricot, $2007: 30$ ).

La compétence médiatique ou compétence informationnelle présente deux aspects. Tout d'abord, pour pouvoir lire, comprendre et exploiter un document qui correspond à ce que l'on cherche, il faut d'abord savoir de quelle manière le trouver. Une fois que l'usager a trouvé le document, un des aspects de la compétence médiatique est d'évaluer la source afin de s'assurer que ce document est pertinent. On peut donc se poser des questions telles que «Qui en est l'auteur? », «Est-il compétent, fiable ? ». Par ailleurs, face à la diversité des sources proposées, le lecteur qui mène une recherche sur le Web se trouve face à différentes sources, "soit potentiellement des points de vue différents, contradictoires » (Tricot, $2007: 67$ ) et il lui est nécessaire d'adopter un point de vue réflexif et critique.

Les individus de manière générale et les jeunes plus particulièrement sont par ailleurs davantage exposés à visionner et à participer à des événements de groupes qui peuvent parfois choquer. C'est pourquoi développer la maitrise de compétences informationnelles telle que la recherche, le traitement et l'évaluation de la source d'information semble aujourd'hui nécessaire, notamment pour les jeunes (Connolly \& McGuinness, 2018 : 85).

\section{Enjeux sociaux et difficultés au quotidien}

Nous devons nous adapter à un environnement professionnel et social qui se transforme. Les démarches se faisant souvent en ligne, la principale difficulté aujourd'hui pour les personnes en situation d'illectronisme est de pouvoir bénéficier de leurs droits.

La seule issue est de développer des stratégies. Selon l'étude CSA Research, les personnes éprouvant des difficultés se tournent donc vers des personnes ressources, la famille, les enfants par exemple ou les amis. Parfois, elles s'appuient sur des repères intuitifs pour certains actes du quotidien comme la mémorisation de signes, pictogrammes ou couleurs. Lorsqu'elles le peuvent, elles intègrent des formations mais on observe combien il peut être difficile d'admettre qu'on a besoin d'être accompagné ou de se former : tout comme la situation d'illettrisme, la situation d'illectronisme isole considérablement les personnes concernées.

Chez les personnes âgées, les difficultés se font plus prégnantes : $41 \%$ des 70 ans et plus n'utilisent jamais Internet. La dématérialisation des démarches administratives peut paraitre compliquée chez les personnes en situation d'illectronisme, qu'elles soient adultes ou seniors, parce que ces démarches sont liées au fait de percevoir des prestations ou des allocations et génèrent des difficultés financières. Les difficultés face au numérique renforcent donc les risques d'exclusion sociale des personnes qui pour certaines sont déjà exclues du fait de leur non-maitrise de la lecture, de l'écriture et du calcul (personnes également en situation d'illettrisme). La Communauté européenne reconnait d'ailleurs depuis 2006 la compétence numérique comme l'une des compétences clés, au même titre que la lecture et l'écriture.

La disqualification professionnelle est l'un des enjeux majeurs liés à la situation d'illectronisme. En effet, les technologies numériques sont aujourd'hui prégnantes au travail, même sur des postes dits peu ou pas qualifiés. 


\section{Focus sur les Digital Natives}

Nés à l'ère du numérique, donc après 1985, les Digital Natives (nom donné par le chercheur américain Mark Prensky) ou encore les "Y» sont supposés maitriser le numérique sans problème parce qu'ils sont nés avec le numérique. Néanmoins, nous constatons, à la lecture de l'enquête réalisée par CSA Research, que bien qu'ils utilisent très souvent les écrans, les jeunes éprouvent des difficultés lorsqu'ils utilisent les outils numériques. Par ailleurs, le terme de Digital Natives ne fait pas l'unanimité parmi les chercheurs ${ }^{4}$. Selon Pascal Plantard (2015), il s'avère que les inégalités chez les adolescents sont également marquées par les territoires : les zones blanches existent à nouveau faute d'entretien d'un certain nombre de points d'accès à Internet. Par ailleurs, les droits des jeunes en ce qui concerne le numérique sont souvent négligés, comme si le numérique ne leur appartenaient pas ou ne les concernaient pas : le numérique est d'ailleurs souvent absent des programmes éducatifs (Connolly \& McGuinness, $2018: 91)$.

Paradoxalement, l'enquête CSA Research montre que les familles de milieux peu favorisés sont très équipées. En effet, les adolescents des familles socialement plus défavorisées possèdent en général un ordinateur fixe ou portable dans leur chambre et même si les jeunes semblent très à l'aise avec les écrans de leurs tablettes et téléphones, on constate des disparités dans l'utilisation du numérique. Selon Nathalie Caclard (2012), on distingue deux catégories chez les jeunes : d'une part, les geeks qui font du codage, d'autre part les jeunes qui utilisent Snapchat mais qui semblent incapables d'envoyer un mail pour postuler à une offre d'emploi (Caclard, 2012 : 22). La médiation numérique reste donc un enjeu majeur même auprès des jeunes.

On peut s'interroger sur ce qui semble poser problème. Concernant l'éducation aux médias, l'équipe de recherche de Pascal Plantard (2015) a constaté que les familles «aisées» considéraient que c'était le rôle des parents d'éduquer aux médias tandis que les familles de milieux défavorisés considéraient que c'était le rôle de l'école. En effet, des études montrent que certains jeunes peuvent rencontrer de vrais problèmes pour trouver des informations fiables et pertinentes et pour s'approprier des méthodes efficaces de recherche sur le Web (Guichard, 2015 : 408). Mais on a également remarqué que les jeunes qui entrent dans leur vie d'adultes éprouvent des difficultés à effectuer leurs démarches administratives ou à utiliser les outils de recherche d'emploi. Au sein d'une même classe d'âge, il existe donc des différences très importantes.

Selon Éric Guichard, on ne peut donc pas affirmer que les Digital Natives possèderaient une véritable culture numérique simplement parce qu'ils sont nés à l'ère du Web. En revanche, formés dans de bonnes conditions, on constate qu'ils acquièrent rapidement cette culture numérique (Guichard, $2015: 409$ ).

\section{Littératie numérique : les pratiques de lecture et d'écriture questionnées}

Bien qu'on ait pu craindre que l'avènement du numérique fasse disparaitre la lecture et l'écriture, il n'en est rien. En s'imposant au sein de nos sociétés, les technologies numériques ont certes modifié nos usages de lecture et d'écriture mais n'en ont pas supprimé l'existence. La lecture sur le Web mobilise néanmoins des caractéristiques cognitives spécifiques. Selon Victor Petit et Serge Bouchardon (Petit \& Bouchardon, 2017: 130), la complexité du numérique a en effet engendré « l'extension du domaine de l'écrit - autant que celui du calcul».

\footnotetext{
${ }^{4}$ Un paragraphe intitulé « Le mythe des "natifs du numérique" » : Connolly N. \& McGuinness C., 2018, Chapitre 7. Vers une littératie numérique pour une participation et une mobilisation active des jeunes dans un monde numérique.
} 


\section{La lecture numérique}

Dans le domaine de la recherche en sciences de l'éducation et en psychologie des apprentissages, on oppose la lecture linéaire des livres à la lecture sur le Web ou sur écran qui est plus «spatiale ». Néanmoins, des travaux de recherche (Amadieu \& Tricot, 2014: 72) démontrent que la lecture d'un document numérique et la lecture d'un document papier mobilisent des compétences de base identiques bien que la lecture sur écran requière également d'autres aptitudes. La lecture numérique diffère de la lecture papier à la fois dans sa structure et dans son contenu (Tricot, 2007 : 66). En effet, dans les textes classiques, il peut s'agir d'une structure narrative ou argumentative mais la structure du texte est connue du lecteur aguerri qui peut s'y repérer. En revanche, un document textuel numérique présente parfois plusieurs structures différentes qui peuvent varier, qui ne sont pas linéaires. André Tricot explique de manière très claire ce phénomène :

Le lecteur élabore une représentation de type $A-B-C$. L'auteur a choisi de présenter $A$, puis $B$, puis $C$. S'il avait voulu écrire $A-C-B$, il l'aurait écrit. Et le lecteur aurait très probablement compris $A-C-B$. Avec les hypertextes, le lecteur se trouve devant la situation suivante : après $A$, il peut aller vers $B$ ou vers $C$. Autrement dit, dans un texte linéaire, il y a une cohérence. Le lecteur à la recherche de cette cohérence peut faire l'hypothèse que l'auteur a écrit son texte d'une certaine manière parce qu'il essayait d'être pertinent. Dans un hypertexte, il y a plusieurs cohérences. (Tricot, 2007 : 111).

Concernant le contenu, il diffère également par la diversité des sources proposées. On peut la définir comme étant pluri-directionnelle. En effet, sur Internet, les informations que nous recherchons se trouvent à droite dans la marge, tout en bas de la page ou sont « invisibles » car apparaissent lorsqu'on clique sur un lien hypertexte dont l'existence est quasiment implicite, à déduire... La lecture est donc composée de différents étages de lecture qui sont matérialisés par les liens hypertextes. Le terme d'hypertexte est apparu dès 1963. Ted Nelson qui l'a le premier utilisé le définit comme étant « un ensemble de documents, où chaque information est stockée une fois, où il n'y a pas de suppression et où chaque information est accessible par un lien à partir de n'importe quel endroit » (Tricot, 2007 : 19-20). Plus précisément, les liens hypertextes apparaissent lorsque l'on passe le curseur dessus (généralement le pointeur de la souris se transforme en main et le lien devient violet lorsqu'il a été activité), qu'un lien hypertexte nous amène à un autre texte. Ce système élaboré en hypertextes propose une nouvelle forme d'organisation de l'information et donc de la lecture. Cet ensemble de textes est beaucoup plus riche qu'un simple texte papier et demande au lecteur d'être davantage impliqué dans sa lecture. En effet, la navigation dans ce système hypertextuel est non linéaire et dépend du choix de chaque individu : chacun clique sur le lien qui lui semble plus pertinent et plus cohérent dans la suite de sa lecture et de se recherche d'informations, contrairement à un texte linéaire où le lecteur n'a pas de choix à faire. Il lui suffit de se laisser guider.

La non-linéarité aurait des effets sur la lecture et sur l'apprentissage car elle représenterait un « surcoût cognitif» (Tricot, 2007 : 63) ou une surcharge cognitive accrue par la vitesse de traitement que nécessite la lecture numérique. La lecture hypertextuelle déstabilise le lecteur et le met «dans un état où il ne sait plus où aller, ni d'où il vient, ni comment y retourner, et surtout, il ne parvient pas à comprendre les contenus qu'il est en train de traiter » (Tricot, 2007 : 64).

Par ailleurs, la mémoire est très sollicitée. En effet, face à la multitude d'informations présentes sur le Web, le lecteur opère un tri afin de conserver les éléments essentiels et pertinents ce qui lui permet de progresser dans la lecture hypertextuelle. Ce tri s'applique presque de manière inconsciente et machinale pour un lecteur aguerri du Web mais c'est moins évident pour un lecteur qui éprouve d'ores et déjà des difficultés avec la lecture papier. 


\section{L’écriture numérique}

Abordons à présent les caractéristiques cognitives sur le plan scriptural.

On observe que les pratiques d'écriture traditionnelles (papier/crayon) sont « manuscrites et latéralisées » et qu'elles sont, tout comme la lecture, caractérisées par leur linéarité. Depuis que le numérique a massivement fait irruption dans notre quotidien, les pratiques scripturales ont évolué : nous écrivons via l'interface d'un écran dans un mode d'écriture " conversationnel ou hypertextuel» (Bros, 2015 : 78-79). On peut définir trois modes d'écriture : les écrits sur traitement de texte (présenter un écrit dans un format numérique), les écrits conversationnels différés (mail par exemple) et les écrits simultanés ou synchrones (tchat).

Lorsque l'on pense écriture numérique, on imagine le traitement de texte et l'écriture dans le contexte de messagerie électronique. Mais d'autres outils numériques mobilisent l'écriture : les messageries instantanées, les $\mathrm{SMS}^{5}$, les outils collaboratifs en ligne... Et on se rend compte par exemple que l'usage des SMS a fait émerger une forme stabilisée de français écrit (Penloup, 2018). En effet, selon Marie-Claude Penloup, cette variété du langage avec ces formes spécifiques simplifiées (remplacement d'un son par une lettre, chiffre utilisé pour sa valeur phonétique...) pourrait devenir des supports permettant de travailler l'écrit avec les jeunes, notamment l'orthographe. Il existe d'autres pratiques d'écriture personnelles numériques comme les $\mathrm{RPG}^{6}$ en ligne ${ }^{7}$ ou les fanfictions qui sollicitent des aptitudes d'écriture et qui permettraient de développer des compétences diverses comme interagir, lire, écrire sur écran.

Que l'on aborde la question de la lecture ou de l'écriture, le numérique a considérablement modifié notre perception de l'écrit. Les repères temporels et spatiaux et les modes relationnels et communicationnels sont différents. La sphère privée et la sphère publique sont à redéfinir progressivement, on le constate dans le cadre de l'usage des réseaux sociaux. Écrire sur soi en ligne pour les amis est devenu courant mais il est important également de savoir configurer les paramètres de confidentialité de son compte au risque de voir sa vie être diffusée à tous.

Le formel et l'informel sont également à repenser dans les modes de communication oraux et écrits (messagerie, logiciel de communication type Skype). Nous sommes encore dans le flou et ces nouveaux modes de relation génèrent des aptitudes à la fois sociales et techniques à acquérir pour les usagers...

\section{Les compétences technologiques : lecture numérique, réponse tactile}

Le numérique aujourd'hui, ce n'est pas seulement l'ordinateur, les tablettes et les smartphones. Selon Alain Dumais (2014), au sein de ce que l'on nomme les outils numériques s'inscrivent également d'autres outils auxquels on pense moins : les guichets et distributeurs automatiques avec des écrans tactiles. De manière générale, ces outils numériques de notre quotidien impliquent des manipulations tactiles et induisent des interactions sensorielles. De nombreux «écrans » ou appareils numériques sollicitent une réponse non pas écrite mais tactile : nous n'écrivons pas, ne saisissons pas de texte sur un clavier mais sélectionnons une réponse parmi une liste. Parfois même l'action impliquée par l'écran digital n'est pas explicitée, elle est implicite : "prendre un ticket »: signifie "toucher l'écran pour éditer un ticket», « actionner une touche » signifie « toucher l'écran ».

\footnotetext{
${ }^{5}$ Short Message Service.

${ }^{6}$ Role Playing Games.

${ }^{7}$ Forums de fans dans lesquels les participants incarnent un personnage d'un univers spécifique.
} 

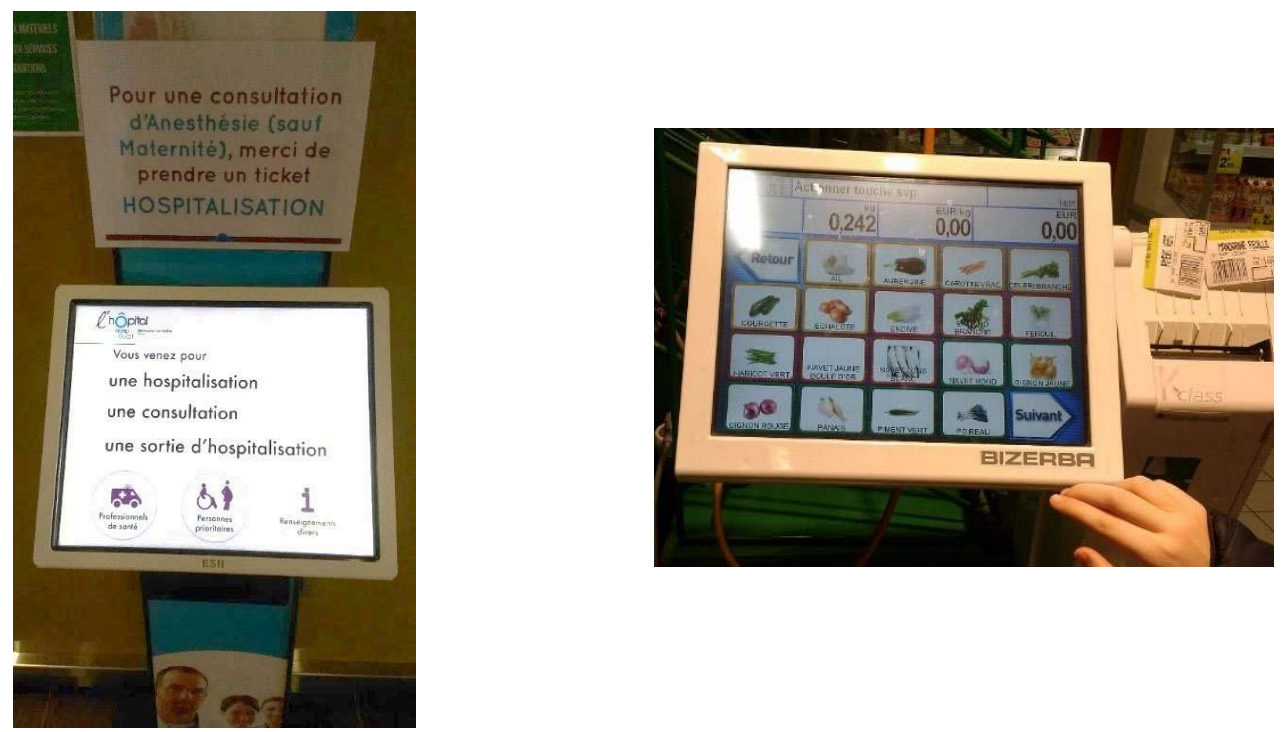

Nous venons de voir que l'usage du numérique préoccupe toutes les personnes, même lorsqu'elles ne se trouvent pas en situation d'illettrisme. Les formes d'exclusion liées à la nonmaitrise de l'écrit sont transposables à la non-maîtrise de l'écrit numérique. Les ateliers de remédiation peuvent permettre d'agir sur la maitrise à la fois de l'écrit et de l'usage des outils numériques.

\section{Les apports du numérique dans l'éducation et la formation}

Avec le numérique, les pratiques de lecture et d'écriture se sont complètement transformées et continuent de changer. La formation initiale et continue des individus s'adapte aujourd'hui aux changements véhiculés par les nouvelles pratiques numériques et peut s'appuyer sur des savoir-faire que chaque individu acquiert en dehors des lieux de formation ou d'éducation.

\section{Utiliser le numérique pour développer d'autres compétences chez les jeunes}

Selon Marie-Claude Penloup (2018), il existe une tendance qui est d'ignorer les pratiques des jeunes hors les murs de l'école mais plutôt réfléchir à une pédagogie enrichie et réinventée grâce au numérique et via les nouveaux formats de temps et d'espace. Par exemple, le langage hybride développé par les SMS pourrait selon elle devenir un matériau d'étude stimulant sur la langue avec les plus jeunes.

Concernant l'éducation aux outils numériques auprès des jeunes, il semble qu'il y ait de nombreuses pistes développées par des chercheurs en science de l'éducation. On a constaté que les jeunes étudiants ou lycéens par exemple utilisent leur smartphone, même au sein des lycées. Les proviseurs d'établissement disent qu'il est compliqué d'obtenir qu'ils ne l'utilisent pas pendant le cours (Plantard, 2015a). Dès qu'ils le peuvent, les lycéens utilisent certaines applications sans difficulté comme Snapchat, Instagram ou Whatsapp. C'est pourquoi, selon Marie-Claude Penloup (2018), pour sensibiliser les jeunes, on peut s'appuyer sur le numérique et s'intéresser à ce qu'ils savent faire. Ces outils peuvent permettre de travailler également l'acquisition de l'écrit ou de la lecture. Selon Marie-Claude Penloup (2018), on observe un effet positif des outils sur tablette qui permettent de guider le geste d'écriture manuscrite, en particulier pour les enfants en difficulté. L'efficacité des outils numériques reste en grande majorité à étudier, mais certaines de leurs fonctions spécifiques semblent pouvoir soutenir les apprentissages. 
Selon Marie-Claude Penloup, grâce aux nouvelles « sollicitations à écrire » offertes par le numérique, les adolescents maitrisent certaines pratiques de l'écrit sur lesquelles l'enseignant peut s'appuyer (Penloup, 2017). Même si tous ne savent pas utiliser les écrans, ils sont nombreux à utiliser les écrans pour écrire, pour texter ou interagir sur des forums (Joannidès, 2014). Les enseignants, les formateurs peuvent s'appuyer sur ces pratiques de lecture et d'écriture pour développer les aptitudes transférables vers le numérique. Un outil numérique peut à la fois être le " poison et le remède », en fonction du lieu et du contexte dans lequel il est utilisé. Il ne faut pas « diaboliser » les outils numériques (2015b) mais plutôt s'en servir (Penloup, 2018).

\section{Changer le regard que les adultes en formation portent sur l'écrit}

Certaines études ont permis de constater que les adultes en formation qui maitrisent mal l'outil scripteur sont parfois plus à l'aise avec l'outil numérique et la saisie au clavier (Bros, 2015). En formation, proposer différents supports et notamment le support numérique pour passer à l'écrit peut permettre de lever des appréhensions. Par ailleurs, le support numérique dans l'apprentissage change la place de l'enseignant/formateur qui devient davantage un accompagnant, un guide. Cela peut atténuer l'appréhension liée au fait de faire une erreur : le formateur favorise une erreur qui devient formative (l'ordinateur ne juge pas) et qui sera moins perçue comme une sanction. L'utilisation de l'outil numérique dans les apprentissages peut également valoriser les connaissances déjà acquises en milieu social. L'enseignant ou le formateur peut les valoriser pour aborder différemment le rapport à l'écrit.

Dans son article, Frédérique Bros (2015: 85) présente le témoignage d'une animatrice d'atelier numérique. Elle a pu constater que l'outil numérique avait permis aux personnes de travailler la lecture et l'écrit sans appréhension. Ils ne considèrent pas la lecture et l'écriture sur le Web comme des pratiques scripturales en tant que telles. En effet, Frédérique Bros précise que les usagers qui évoluent sur le Web an cliquant sur les liens hypertextes, qui passent d'un lien à l'autre, d'une page à l'autre, qui remplissent des formulaires, cliquant, cochant n'ont pas la sensation d'effectuer des activités liées à la lecture ou à l'écriture. L'animatrice de cet atelier numérique constate les outils numériques ne suscitent pas la même appréhension que l'utilisation du papier et du crayon. L'outil numérique permettrait donc d'atténuer le sentiment d'insécurité lié à l'écrit chez des adultes en formation.

\section{De nouvelles pratiques d'expression écrite}

Par ailleurs, le développement des outils numériques fait émerger des pratiques personnelles d'écriture. On constate que ces pratiques sont devenues très courantes dans les sociétés contemporaines. D'une part, l'usage du numérique implique la pratique de l'écriture via par exemple les réseaux sociaux ou les blogs. Mais de nouvelles formes de sociabilité développent également de nombreuses formes d'expression sur le Web. On peut citer notamment la créativité et l'écriture libre des individus. Des enquêtes sur les pratiques culturelles des Français, des Anglais et des Américains démontrent qu'aujourd'hui, en moyenne un individu sur deux exerce une activité d'écriture créative via le numérique (Cardon, 2019: 189). On constate notamment que la création d'un blog suscite des activités d'écriture libres et créatives où les personnes peuvent partager leurs loisirs et leurs activités personnelles (photo, cuisine, littérature, couture, cinéma, maquettes...). Les technologies se sont simplifiées et permettent aujourd'hui via des tutoriels disponibles sur le Web de réaliser des montages des vidéos par exemple. Ces pratiques principalement amateurs pourraient être considérées par les formateurs et enseignants comme un levier pédagogique pour développer à la fois les pratiques de l'écrit et les pratiques numériques, les deux fonctionnant de manières transversales (Cardon, 2019 : 192). 


\section{Une action de formation pour développer la maitrise de l'écrit et du numérique}

\section{Méthodologie et cadre d'analyse}

L'étude que nous présentons dans cet article a été menée en 2018 sur un groupe de stagiaires en formation professionnelle "écrit et numérique " au sein d'un organisme de formation lyonnais. L'action de formation proposée était un dispositif destiné à huit personnes salariées en situation d'illettrisme ou d'analphabétisme. Les personnes étaient affectées à des postes peu ou pas qualifiés : peintre en bâtiment, agent des espaces verts, couturier, agent de nettoyage. Les niveaux d'étude étaient variables mais de manière générale, il s'agissait de personnes n'ayant pas suivi d'études longues. Trois d'entre elles étaient sorties du système scolaire en fin de collège, trois avaient validés un CAP ou BEP, une personne avait obtenu son baccalauréat et une autre avait suivi des études supérieures pendant deux ans sans valider de diplôme universitaire. Les participants, quatre hommes et quatre femmes, étaient âgés de 23 à 57 ans.

Les objectifs de la formation étaient de travailler à la fois sur l'écrit et sur les outils numériques utiles au quotidien. En effet, l'employeur de ces participants avait remarqué que ces personnes avaient besoin d'un accompagnement à la fois dans la production d'écrit dans le cadre professionnel mais également dans leur vie personnelle, dans la gestion de l'écrit numérique lié aux démarches administratives. L'objectif était donc de leur permettre d'acquérir davantage d'autonomie dans la vie quotidienne, dans la recherche d'un emploi pérenne. Les personnes participaient à des séances « écrit et numérique » au sein de l'organisme de formation deux demi-journées par semaine et demeuraient à leur poste de travail le reste de la semaine.

Notre projet a été de voir si cette formation pouvait être bénéfique et leur apporter effectivement davantage d'autonomie. Les contenus de la formation se sont adossés aux constats de la société telle qu'elle existe aujourd'hui : une société de l'écrit et où le numérique est de plus en plus présent notamment pour les démarches administratives. En début de parcours formatif, les contenus ont été définis en collaboration avec les stagiaires, formalisés par un contrat de formation lors d'un entretien d'accueil et de pré-positionnement au cours duquel les personnes ont pu faire part librement de leurs besoins et de leurs attentes. Dans ce parcours de formation, il était prévu que les contenus des séances s'adapteraient par la suite et seraient ajustés en fonction des besoins formulés des stagiaires et de leurs demandes concrètes.

Chaque séance de formation était donc liée à un objectif concret et une application pratique. Au cours de cette formation, les stagiaires ont appris à utiliser les différentes fonctions du traitement de texte et ont ainsi pu élaborer leur Curriculum Vitae ou effectuer des recherches sur le Web afin de sélectionner une liste d'entreprises au sein desquelles ils pourraient envoyer une candidature. Plus précisément, les activités développaient l'acquisition à la fois de l'écrit et des usages du numérique, comme par exemple la création d'une messagerie électronique pour rédiger un mail à un membre de la famille ou un ami mais également à un recruteur (avec envoi de Curriculum Vitae en pièce jointe) en travaillant sur les différents types d'écrit, familier et formel. Ils ont également abordé les enjeux des réseaux sociaux à travers des usages différenciés (inscription à des réseaux sociaux professionnels par exemple). Certains étant jeunes parents, apprendre à se méfier des addictions liées aux écrans et savoir accompagner leurs enfants faisait aussi partie du programme de leur formation.

L'écrit n'était jamais travaillé « frontalement ». Les activités pédagogiques étaient abordées en contexte via un outil numérique favorisant la collaboration, l'échange et l'autonomie. Par exemple, la création d'une charte de vie du groupe sur un padlet ou l'utilisation des réseaux sociaux pour communiquer entre eux a favorisé la créativité et l'interactivité des participants. Les formateurs ont privilégié l'utilisation de supports numériques variés : ordinateur, tablettes mais également smartphones. Les stagiaires avaient besoin de se connecter, de s'identifier dans le cadre des démarches administratives. Ils ont donc appris à utiliser les paramètres de confidentialité. L'utilisation du smartphone et des SMS, largement répandu surtout chez les 
jeunes stagiaires, répondait à des besoins évoqués dans un processus de socialisation. Intégré naturellement lors des séances a priori vouées à l'utilisation des outils numériques, l'écrit était complètement présent mais sans jamais susciter l'appréhension de la part des stagiaires.

\section{Résultats de cette étude}

Nous avons analysé différents paramètres au cours de la formation pour vérifier si ce type de formation pouvait s'avérer efficace.

Nous avons tout d'abord pris en compte l'assiduité du stagiaire en formation qui permet de mesurer l'investissement. Nous avons également observé les demandes exprimées au formateur sur des besoins concrets à l'écrit, sur les usages numériques ou sur les deux domaines. Ces demandes d'aide étaient recensées sur un document individuel pour chaque stagiaire. Enfin, nous avons mesuré les progrès effectués en fin de formation, d'abord par le biais d'une évaluation de fin de parcours qui abordait les deux aspects : langue écrite et habileté numérique, puis par le biais de témoignages écrits par les apprenants à l'issue de la formation.

L'observation de la présence en formation a montré des résultats positifs: sur les huit participants, tous sont allés au terme du parcours. Deux d'entre eux ont éprouvé des difficultés à se mobiliser sur les apprentissages écrits et ont été absents lors de quelques séances mais ils ont malgré tout terminé la formation. Concernant les demandes d'accompagnement individuelles, six personnes sur huit en ont fait usage. Les demandes étaient très variables : un accompagnement à la déclaration de revenus en ligne, une demande d'aide pour installer et prendre en main un ordinateur portable récemment acquis, un accompagnement à la recherche d'emploi (rédaction de CV, lettre de motivation...), un besoin spécifique pour la déclaration de ressources à la CAF ou pour la lecture de document juridique.

L'analyse des évaluations et des témoignages a permis de révéler que les huit stagiaires ont acquis la maitrise de la messagerie électronique avec envoi de mail pour des candidatures ou pour demander des rendez-vous (notamment aux accompagnants sociaux ou référents insertion), la capacité à rechercher et trier des informations sur le Web ou d'utiliser les outils numériques pour s'orienter dans la ville.

Dans le cadre de cette démarche, concernant l'écrit et le numérique, ce qui apparait de manière moins mesurable, c'est l'acquisition d'une certaine autonomie dans les démarches administratives ou les achats en ligne. Les formateurs ont en effet remarqué une prise de confiance qui s'est matérialisée en dehors des heures de formation par l'envoi de mails pour prévenir d'une absence ou pour transmettre un Curriculum Vitae à un recruteur. Au cours de la formation, les stagiaires devenaient de plus en plus autonomes dans les démarches et les échanges avec les administrations. Par exemple, en fin de formation, ils étaient en mesure d'actualiser leur situation sur le site de Pôle Emploi ou d'effectuer leur déclaration d'activité sur le site de la Caisse d'Allocation Familiale sans être accompagnés.

On peut donc supposer que cette action de formation a pu permettre d'accompagner ces participants dans une démarche d'autonomisation ayant pour incidence d'améliorer leur insertion socioprofessionnelle.

\section{Bibliographie}

Amadieu F., \& Tricot A., 2014, Apprendre avec le numérique : Mythes et réalités, Paris : Retz. Bros F., 2015, « Devenir e-lettré : Quels leviers et voies d'accès à l'écrit à l'heure de la littératie numérique ? », Savoirs, N³8 (2), 73-90.

Caclard N., 2012, « La médiation numérique : Une urgence pédagogique et politique », Cahiers de l'action, $\mathrm{n}^{\circ} 36$ (2), 21-25. 
Cardon D., 2019, Culture numérique, Paris : Presses de Sciences Po.

Connolly N., \& McGuinness C., 2018, "Chapitre 7. Vers une littératie numérique pour une participation et une mobilisation active des jeunes dans un monde numérique », In Points de vue sur la jeunesse - Les jeunes à l'heure du numérique (Vol. 4). Strasbourg : Conseil de l'Europe.

Fastrez P., 2012, Translittératie et compétences médiatiques. La translittératie en débat: regards croisés des cultures de l'information (infodoc, médias, informatique) et des disciplines, Rennes.

Guichard É., 2015, «Culture numérique, culture de l'écrit », Interfaces numériques, nº (3), 403-420.

Joannidès R., 2014, L'écriture électronique des collégiens: Quelles questions pour la didactique $d u$ français?, Thèse de doctorat soutenue à l'Université de Rouen Normandie, France.

Mazet P., 2019, « Vers l'État plateforme : La dématérialisation de la relation administrative », Revue en ligne La vie des idées.

OCDE, 2000, La littératie à l'ère de l'information [Rapport final de l'Enquête internationale sur la littératie des adultes], Canada: OECD Publishing.

Penloup M.-C., 2017, «Didactique de l'écriture: Le déjà-là des pratiques d'écriture numérique », Le français aujourd'hui, $\mathrm{n}^{\circ} 196$ (1), 57-70.

Penloup M.-C., 2018, «Peut-on mobiliser les pratiques hors école de l'écriture numérique? Littératie numérique extrascolaire et enseignement du français », Conférence Consensus : Paris.

Petit V., \& Bouchardon S., 2017, «L'écriture numérique ou l'écriture selon les machines. Enjeux philosophiques et pédagogiques », Communication langages, $\mathrm{n}^{\circ} 191$ (1), 129148.

Portnoff A.-Y., 2019, «Les Français et le numérique. Les enseignements du Baromètre du numérique 2018 du CREDOC », Futuribles, n429 (2), 33-42.

Rosa H., 2010, Accélération: Une critique sociale du temps, Paris : La Découverte.

Tricot A., 2007, Apprentissages et documents numériques, Paris : Belin. 


\section{GLOTTOPOL}

Revue de sociolinguistique en ligne

Comité de rédaction : Michaël Abecassis, Salih Akin, Sophie Babault, Claude Caitucoli, Véronique Castellotti, Régine Delamotte, Robert Fournier, Stéphanie Galligani, Emmanuelle Huver, Normand Labrie, Foued Laroussi, Benoit Leblanc, Fabienne Leconte, Gudrun Ledegen, Danièle Moore, Clara Mortamet, Alioune Ndao, Isabelle Pierozak, Gisèle Prignitz.

Rédactrice en chef : Clara Mortamet.

Comité scientifique : Claudine Bavoux, Michel Beniamino, Jacqueline Billiez, Philippe Blanchet, Pierre Bouchard, Ahmed Boukous, Pierre Dumont, Jean-Michel Eloy, Françoise Gadet, Monica Heller, Caroline Juilliard, Jean-Marie Klinkenberg, Jean Le Du, Marinette Matthey, Jacques Maurais, Marie-Louise Moreau, Robert Nicolaï, Didier de Robillard, Paul Siblot, Claude Truchot, Daniel Véronique.

\section{Comité de lecture pour ce numéro :}

Mickael Abecassis, Michelle Auzanneau, Salih Akin, Céline Amourette, Gabriel Bergounioux, Philippe Boula de Mareuil, Catherine Brissaud, Aude Bretegnier, Maria Candea, Véronique Castellotti, Régine Delamotte, Jean-François De Pietro, Catherine Delarue-Breton, Anne Dister, Alexandre Duchêne, Valentin Feussi, Françoise Gadet, Stéphanie Galligani, François Gaudin, Monica Heller, Emmanuelle Huver, Caroline Juilliard, Abou Bakri Kebe, Jean-Marie Klinkenberg, Christian Lagarde, Foued Laroussi, Fabienne Leconte, Evelyne Lloze, Nolwenn Lorenzi, Gudrun Ledegen, Nadja Maillard, Marinette Matthey, Bruno Maurer, Véronique Miguel Addisu, Saskia Mugnier, Claire Oger, Marielle Rispail, Richard Sabria, Véronique Traverso, Christel Troncy, Cécile Van den Avenne. 